\title{
GRAVIDEZ E MATERNIDADE DE ADOLESCENTES: FATORES DE RISCO E DE PROTEÇÃO
}

\section{PREGNANCY AND MATERNITY OF ADOLESCENTS: RISK AND PROTECTOR FACTORS}

\author{
Nancy Ramacciotti de Oliveira-Monteiro ${ }^{1,3}$ \\ Mariana Negri ${ }^{1}$ \\ Amanda Oliveira Fernandes ${ }^{1,3}$ \\ Juliana Olivetti Guimarães Nascimento ${ }^{2,3}$ \\ Fábio Tadeu Montesano 1,3
}

Oliveira-Monteiro NR et al. Gravidez e maternidade de adolescentes: fatores de risco e de proteção. Rev Bras Cresc e Desenv Hum 2011; 21(2): 198-209.

\section{RESUMO}

Introdução: a procriação na adolescência é parte das preocupações relativas à saúde e desenvolvimento de jovens, a despeito de seu significado não necessariamente negativo.

Objetivo: o estudo teve como objetivo avaliar competência, problemas psicológicos e indicativos de fatores de risco e de proteção ao desenvolvimento num grupo de adolescentes grávidas e mães. Método: com uso do Youth Self Report (YSR) e da Escala de fatores de proteção/risco na maternidade adolescente (FRIP), foram pesquisadas 46 adolescentes de 13 a 18 anos, usuárias de serviços públicos de saúde da Baixada Santista: 27 gestantes primigestas e 19 mães primíparas com filhos menores de um ano. Resultado: níveis de competência foram menores nas adolescentes gestantes e mães mais pobres. A pobreza não ficou associada a indicadores de problemas psicológicos, que foram avaliados na amostra com tendência à faixa de normalidade. As mães apresentaram referências de qualidades pessoais em níveis significativamente maiores do que as gestantes. Esperanças e sonhos para a própria vida além do contato positivo com a família e com o pai da criança foram fatores de proteção apontados, enquanto proximidade de moradia ao tráfico de entorpecentes foi o principal fator de risco identificado. Conclusão: fatores de proteção nos contextos ambientais das adolescentes pesquisadas podem estar interrelacionados ao desenvolvimento da competência, importante recurso frente à situação da maternidade de adolescentes.

Palavras-chave: gravidez na adolescência; fatores de risco e proteção; desenvolvimento humano.

1 Laboratório de Psicologia Ambiental e Desenvolvimento Humano - LADH/ UNIFESP - Campus Baixada Santista. Avenida Ana Costa, 95 - Vila Mathias - Santos/SP - CEP:11060-001. Departamento: Saúde, Educação e Sociedade

2 Universidade Federal de São Paulo - UNIFESP - Campus São Paulo. R. Borges Lagoa, 570 - $1^{\circ}$ andar - Vila Clementino/SP 04038-030. Programa de Pós-Graduação em Psiquatria e Psicologia Médica

3 Universidade Federal de São Paulo - UNIFESP - Campus Baixada Santista

Correspondência para: Nancy Ramacciotti de Oliveira-Monteiro. Universidade Federal de São Paulo - UNIFESP - Campus Baixada Santista - Avenida Ana Costa, 95 - Vila Mathias - Santos/SP - 11060-001. E-mail: nancy.unifesp@gmail.com

Órgão de Fomento: Conselho Nacional de Desenvolvimento Científico e Tecnológico (CNPq) (Proc. CNPq 114924/2008 2), vinculado ao Programa Institucional de Bolsa de Iniciação Científica (PIBIC) da Universidade Federal de São Paulo (UNIFESP). 


\begin{abstract}
Introduction: breeding in adolescence is among the concerns related to youth development and health, not necessarily with a negative significance. Objective: the present study researched competence data, psychological problems and indicative of risk and protection factors inside a group of pregnant teenagers and teen mothers. Method: for that purpose, the Youth Self Report (YSR) and a scale of risk/protection factors during teen maternity (FRIP) were used. We surveyed 46 adolescents between 13 and 18 years, users of health services offered in Santos (SP) region: 27 at first pregnancy and 19 with firstborns under one year of age. Results: the level of competence was lower among poorer adolescents, were they pregnant or mothers. Poverty was not associated with indicators of psychological problems, which were evaluated within a normality range by the YSR. Mothers presented self reference of personal qualities in levels significantly higher than the pregnant girls. Risk / protection factors involving environmental systems were indicated. Claims of hope and dreams about their own lives besides positive contacts with their families and with the fathers of their children were appointed as protection factors, while the proximity of their households to the traffic of drugs was the main risk factor identified. Conclusion: $p$ rotection factors derived from the environmental contextures of the adolescents researched might be related to the development of the competence, an important resource regarding the situation of adolescent motherhood.
\end{abstract}

Key words: pregnancy in adolescence, risk and protection factors; human development.

\section{INTRODUÇÃO}

A gravidez na adolescência é tema controverso no âmbito da saúde sexual e reprodutiva ${ }^{1}$, e preocupação presente em organismos nacionais e internacionais dedicados ao cuidado, saúde e desenvolvimento de jovens. Por sua multidimensionalidade, o tema traz desafios para os profissionais de saúde, educadores, governo e sociedade em geral. Segundo dados da Organização Mundial de Saúde (OMS)², o Brasil, em conjunto com Bangladesh, Congo, Etiópia, India, Nigéria e Estados Unidos, realizam metade dos partos de adolescentes que ocorrem em todo mundo.

Estudos sobre gravidez e maternidade de adolescentes brasileiras vêm indicando a heterogeneidade do fenômeno da procriação durante a segunda década de vida, destacando suas dimensões psicossociais e a multicausalidade de fatores envolvidos ${ }^{3-6}$. A estrutura familiar, $o$ nível econômico, a escolaridade, a relação com o pai da criança, a inserção em contextos de violência e de tráfico de entorpecentes são exemplos de dimensões constitutivas para a problemática da gravidez de adolescentes no tocante a problemas de desenvolvimento da adolescente e de seu filho?

A gravidez na adolescência alcança muitos e diferentes âmbitos do desenvolvimento psicossocial da jovem ao modificar formas de relacionamento com seus familiares, com o pai da criança, em seus vários contextos ambientais, e consigo mesma - mudanças não necessariamente negativas ${ }^{8}$.

Significados positivos da gravidez e da maternidade na adolescência parecem presentes para algumas adolescentes ao manifestarem desejos de engravidar e parecerem orgulhar-se de serem mães, seja por se sentirem fortalecidas pela própria fertilidade, mostrarem maior autonomia perante adultos, ou mesmo libertaramse de situações ambientais adversas ${ }^{3-4}$. 
Há sugestão de que mães adolescentes apresentam mais problemas de saúde mental que mães adultas 9 . Por outro lado, há indicadores de que adolescentes que apresentam problemas psicológicos são mais sujeitos à maternidade e paternidade ${ }^{10}$. Sapienza e Pedromônico ${ }^{11}$ apontam que muitas variáveis podem ser consideradas associadas a fatores de risco presentes na infância e na adolescência como depressão, ansiedade, estresse, distúrbios de conduta, evasão escolar, problemas de aprendizagem, uso de drogas, violência familiar, desagregação familiar, violência física, abandono, maus-tratos, e gestação precoce.

A temática relativa a fatores de proteção diante de condições de vulnerabilidade vem sendo tratada com respeito à saúde integral de adolescentes ${ }^{12}$. Fatores de proteção são aquelas condições que moderam a relação entre os riscos e o desenvolvimento do sujeito, como influências que modificam, melhoram ou alteram respostas pessoais a determinados riscos. Um evento negativo de vida poderá ser considerado como fator de risco quando sua presença aumentar a probabilidade de provocar problemas físicos, sociais ou emocionais ${ }^{13}$.

Os fatores protetivos moderadores, que podem pertencer a diferentes níveis ambientais, modificam respostas de pessoas aos fatores de risco, num sentido adaptativo, como no caso de estratégias de competência e comportamentos pró-sociais ${ }^{14-15}$, e de resiliência ${ }^{16}$. Também recursos internos dos indivíduos, diferentes níveis de coesão ecológica no ambiente do qual fazem parte, e a rede de apoio social e afetiva podem auxiliá-los no enfrentamento de adversidades.

Nessa linha de pensamento, estudos de Oliveira-Monteiro ${ }^{3-4}$ e Cerqueira-Santos ${ }^{17}$ indicam que a gravidez na adolescência não pode por si só ser considerada fator de risco enquanto fenômeno diversificado por sua inserção em contextos ambientais e condições pessoais específicas.
O conceito de desenvolvimento humano, para Bronfenbrenner ${ }^{18}$, relaciona-se com um conjunto de processos pelos quais as particularidades da pessoa e do ambiente interagem para produzir constância e mudança nas características da pessoa no curso de sua vida. Trata-se de uma mudança na maneira pela qual uma pessoa percebe e lida com seu ambiente. Segundo esse autor, durante o desenvolvimento ocorrem aquisições de conhecimentos, habilidades e capacidades para conduzir e direcionar o próprio comportamento através de situações e domínios evolutivos, tanto isoladamente como através de uma combinação entre eles (domínios: intelectual, físico, sócioemocional, motivacional e artístico).

As disfunções de desenvolvimento seriam manifestações recorrentes de dificuldades em manter o controle e a integração do comportamento através de situações em diferentes domínios. Competências e disfunções emergeriam de interconexões ambientais e de seu impacto sobre as forças que afetam o crescimento psicológico, dentro do meio ambiente (chamado de ecológico).

A teoria ecológica do desenvolvimento humano ${ }^{18}$ estabelece como ambiente ecológico a interação de quatro níveis ambientais que, interconectados, funcionam como sistemas encaixados, todos recebendo influência/influenciando da/a pessoa em desenvolvimento - os sistemas: micro, meso, exo, macro.

O sistema micro refere-se ao ambiente imediato (nas relações face a face estáveis); o sistema meso é um conjunto de microssistemas (incluindo vizinhos, parentes, escola, bairro), uma inter-relação de dois ou mais ambientes, nos quais a pessoa/família participa ativamente; no exossistema, um ou mais membros da família têm relações face a face, de forma que existe uma interação indireta com outros membros da família não presentes (como a rede de apoio social, a comunidade, e o trabalho); e o sistema macro, que é mais amplo, inclui o padrão global de ideologias, valores e crenças pró- 
prios de uma cultura, que tem reflexo direto no desenvolvimento humano ${ }^{18-20}$.

O conceito de desenvolvimento global da pessoa envolve então a noção de competências, em especial, de competências sociais. A competência social é definida como um conjunto de habilidades sócio-cognitivas e de conhecimento (incluindo a capacidade de controle emocional) que mediam comportamentos específicos, julgados como comportamentos efetivos e idôneos, por outras pessoas e pelo próprio sujeito. O desenvolvimento dessas competências sociais contribui para o desenvolvimento de um adequado ajuste social ${ }^{21}$. A competência social engloba processos complexos, habilidades e estratégias de diferentes dimensões, e é componente chave da personalidade saudável e feliz ${ }^{22}$.

Por outro lado, durante o desenvolvimento humano podem acontecer problemas de ordem psicológica ou comportamental. Achenbach e Edelbrock ${ }^{23}$ classificam os problemas psicológicos e comportamentais de adolescentes em três grupos: 1) de abuso de substâncias; 2) problemas internalizantes (manifestados por perturbações emocionais e cognitivas); e 3) problemas externalizantes (problemas comportamentais ou de atuação, como nos comportamentos antissociais).

Em trabalho sobre adolescentes brasileiros, Dell'Aglio e Hutz ${ }^{24}$ informam que, em 2003, a prevalência de depressão maior em adolescentes foi $3,3 \%$ a $12,4 \%$, enquanto que na população em geral, a prevalência variava em torno de $4 \%$ a 10\%. Segundo estudo de Rocha e Silvares ${ }^{25}$, a competência em adolescentes pode ser fator de proteção diante desses problemas psicológicos e de comportamento.

Com utilização de parâmetros da teoria ecológica de desenvolvimento, apresenta-se este estudo que teve como objetivo avaliar competência, problemas psicológicos e indicativos de fatores de risco e de proteção ao desenvolvimento, num grupo de adolescentes grávidas e mães.

\section{MÉTODO}

Amostra: foram investigadas 46 adolescentes, com idades de 13 a 18 anos, usuárias de serviços públicos de saúde de municípios da Baixada Santista (SP), sendo 27 gestantes primigestas (de idades gestacionais diferentes) e 19 mães primíparas, com filhos menores de um ano. O Critério Brasil ${ }^{26}$ foi utilizado para caracterização socioeconômica da amostra que apresentou indicadores de $66 \%$ das gestantes e $84 \%$ das mães dentre as classes econômicas $\mathrm{C}$ e $\mathrm{D}$ (mais pobres). Na época dos trabalhos de campo, $52 \%$ das adolescentes gestantes e $47 \%$ das adolescentes mães referiram estar morando com suas famílias de origem.

Aspectos éticos: o estudo seguiu procedimentos que garantiram cuidados éticos pertinentes à pesquisa com seres humanos. O projeto foi aprovado pelo Comitê de Ética em Pesquisa da Universidade Federal de São Paulo (CEP/Unifesp - nº1527/08).

\section{INSTRUMENTOS}

I. O "Inventário de Auto Avaliação para jovens" (Youth Self-Report - YSR - for ages 1118), de Achenbach e Rescoria ${ }^{27}$ fornece uma auto avaliação de comportamentos feita por adolescentes. O YSR encontra-se em processo de validação brasileira, contando com índices psicométricos preliminares ${ }^{28}$, sendo utilizado por pesquisadores brasileiros, especialmente a partir dos anos $2000^{25,29-32}$.

O YSR propicia indicadores de dois níveis em itens relacionados com competência e com problemas de comportamento elou psicológicos. O primeiro nível engloba três escalas de competência (atividades, social e desempenho acadêmico) que geram a escala total de competências, apreciando amizades, responsabilidades, participação em grupos, tarefas realizadas habitualmente, esportes, passatempos e desempenho acadêmico. O segundo nível do 
inventário, dos problemas de comportamento, inclui escalas de internalização, externalização e total de problemas de comportamento, permitindo apreciar indicadores autorreferidos de problemas de comportamento e emocionais (como ansiedade/depressão, isolamento/depressão, queixas somáticas, problemas com o contato social, com pensamento e atenção, com violação de regras, além de comportamento agressivo).

II. Uma escala de fatores de proteção/ risco para maternidade de adolescentes (FRIP) foi proposta e utilizada para exploração de fatores de risco e de proteção envolvidos no desenvolvimento das adolescentes grávidas e mães. $\mathrm{O}$ instrumento consiste numa escala do tipo Likert, com 34 afirmações numeradas com 0 (nunca), 1 (quase nunca), 2 (às vezes), 3 (frequentemente) e 4 (sempre), e mais uma questão aberta. Todas as afirmações podem ser relacionadas a fatores de risco ou de proteção, em situações pessoais e contextuais/ambientais de adolescentes, sendo 23 delas referentes a fatores de proteção e 11 a fatores de risco.

A escala foi desenvolvida considerando resultados de pesquisas com adolescentes grávidas e mães usuárias de serviços públicos de saúde e pertencentes a periferias sociais e urbanas de cidades paulistas de médio porte ${ }^{3-4} \mathrm{e}$ também referências da literatura sobre fatores de risco e de proteção, e sobre resiliência ${ }^{15-17,33-}$ ${ }^{35}$. Respondidos de acordo com o ponto de vista da própria adolescente gestante/mãe, os itens da escala incluem afirmações sobre sua pessoa, sua vida e sobre alguns dos seus ambientes cotidianos.

A graduação (de zero a 4) das afirmações da escala FRIP possibilita a indicação de fatores de proteção ou de fatores de risco, de acordo com a semântica de cada afirmação e da valoração escolhida. A avaliação da presença de fator de proteção ou de risco fica indicada pela gradação 3 (frequentemente) e 4 (sempre), nos 23 itens afirmativos para fator de proteção, e nos 11 itens afirmativos para fator de risco. A escala permite uma sistematização a partir do proposto pela teoria ecológica do desenvolvimento humano ${ }^{18}$, no que diz respeito ao modelo dos contextos ambientais interconectados (micro, meso, exo e macro sistemas).

Procedimentos de análise dos dados: os dados do YSR foram transcritos para o software de correção do inventário (Assessment Data Manager Program - ADM; YSR, versão 2001), desenvolvido pela equipe do Sistema de Avaliação Baseado em Evidências do Achenbach (ASEBA) para esse fim. O programa permite apresentar os escores nos níveis: normal, limite e clínico. Além da análise descritiva foi feita uma comparação dos subgrupos de adolescentes gestantes e mães, em relação ao YSR com uso do modelo de análise de variância com dois fatores fixos.

Os resultados da escala FRIP foram obtidos de maneira descritiva, por frequência das respostas. Para descrever os itens da escala FRIP, as respostas frequentemente (pontuação 3) e sempre (pontuação 4) foram consideradas como indicativos de presença de fatores de proteção ou de risco.

\section{RESULTADOS}

Os resultados do YSR indicaram escores dentro da normalidade (acima de 30), tanto das adolescentes gestantes como mães, para a escala Social do nível de Competência. A escala de Atividades apresentou níveis dentro da faixa normal para as gestantes, e limítrofe (escores entre 30 e 35) para as mães.

A média dos escores da Escala Total de Competências apresentou-se dentro da faixa limítrofe, tanto para gestantes como para as mães, resultado de baixos escores de Desempenho Acadêmico obtidos pelo grupo.

A Tabela 1 apresenta escores médios e desvios-padrão relativos à Competência autorreferidas pelas adolescentes gestantes e mães, em seus 
componentes Atividades, Social e Desempenho acadêmico, além do Total de Competências.

Tabela 1: Medidas descritivas das escalas de competências do YSR, segundo situação, na amostra estudada

\begin{tabular}{lrrrr}
\hline & \multicolumn{2}{c}{ Gestante } & Mãe & \\
& Média & DP & Média & DP \\
\hline Atividades & 36,00 & 10,68 & 33,58 & 6,81 \\
Social & 35,30 & 7,18 & 38,32 & 8,43 \\
$\begin{array}{l}\text { Desempenho } \\
\text { acadêmico }\end{array}$ & 1,93 & 0,43 & 1,88 & 0,46 \\
$\begin{array}{l}\text { Total de } \\
\text { competências }\end{array}$ & 32,00 & 8,82 & 31,77 & 6,53 \\
\hline
\end{tabular}

A média do Total de Competências das adolescentes investigadas (gestantes e mães) foi maior naquelas adolescentes pertencentes às classes econômicas $\mathrm{B} 1$ ou $\mathrm{B} 2$ do Critério Brasil ${ }^{26}$, comparadas com adolescentes das classes econômicas $\mathrm{C}$ ou D $(\mathrm{p}=0,014)$. Na Tabela 2 estão apresentados resultados relativos à Competência autorreferida, considerando níveis socieconômicos dos subgrupos investigados, através do Critério Brasi126.

Tabela 2: Medidas descritivas das escalas de competências do YSR, segundo Classe Econômica, na amostra estudada

\begin{tabular}{lrrrc}
\hline & \multicolumn{2}{c}{ B1 ou B2 } & \multicolumn{2}{c}{ C ou D } \\
& Média & \multicolumn{1}{c}{ DP } & Média & DP \\
\hline Atividades & 39,50 & 10,70 & 33,41 & 8,31 \\
Social & 40,33 & 8,17 & 35,21 & 7,29 \\
$\begin{array}{l}\text { Desempenho } \\
\text { acadêmico }\end{array}$ & 2,00 & 0,00 & 1,88 & 0,53 \\
$\begin{array}{l}\text { Total de } \\
\text { competências }\end{array}$ & 36,82 & 9,54 & 29,76 & 6,23 \\
\hline
\end{tabular}

Quanto às áreas de Problemas de Comportamento indicadas pelo YSR, os dados da amostra tenderam a uma distribuição na faixa da normalidade (escores abaixo de 60). A autorreferência de Problemas Externalizantes apresentou indicadores de faixa limítrofe para as adolescentes mães investigadas (um pouco acima de escore 60). A Tabela 3 apresenta resultados relativos a Problemas
Internalizantes e Problemas Externalizantes, além do Total de Problemas indicados no YSR nos subgrupos.

Tabela 3: Medidas descritivas das escalas de competências do YSR, segundo situação, na amostra estudada

\begin{tabular}{lcccc}
\hline & \multicolumn{2}{c}{ Gestante } & Mãe & \\
& Média & DP & Média & DP \\
\hline $\begin{array}{l}\text { Problemas } \\
\text { internalizantes }\end{array}$ & 58,26 & 9,14 & 56,21 & 5,37, \\
\hline $\begin{array}{l}\text { Problemas } \\
\text { externalizantes }\end{array}$ & 59,96 & 8,38 & 60,63 & 9,05 \\
$\begin{array}{l}\text { Total de } \\
\text { Problemas }\end{array}$ & 58,78 & 8,86 & 58,79 & 8,71 \\
\hline
\end{tabular}

Para comparar as adolescentes mães e gestantes com relação a cada uma das variáveis autorreferidas no YSR foi empregado o teste $\mathrm{t}$ de Student para amostras não relacionadas.

A Tabela 4 apresenta níveis descritivos dos resultados comparativos relativos à competências, problemas e qualidades autorreferidas. Esses resultados permitem afirmar que as adolescentes investigadas diferem quanto à variável Qualidades positivas, para a qual as adolescentes mães, em média, apresentaram 6,7 pontos a mais do que as gestantes.

Tabela 4: Resultados da comparação entre os subgrupos com relação a variáveis do YSR

\begin{tabular}{lc}
\hline \multicolumn{1}{c}{ Variável } & Nível descritivo \\
\hline Atividades & 0,377 \\
Social & 0,177 \\
Desempenho acadêmico & 0,753 \\
Total de competências & 0,930 \\
Problemas Internalizantes & 0,393 \\
Problemas Externalizantes & 0,780 \\
Total de Problemas & 0,996 \\
Qualidades positivas & 0,019 \\
\hline
\end{tabular}

Resultados da escala FRIP indicativos de fatores de risco e de fatores de proteção estão nas Tabelas 5 e 6 apresentados por 
Tabela 5: Percentuais Fatores de Risco na Escala FRIP

\begin{tabular}{lcc}
\hline \multicolumn{1}{c}{ Tema } & $\mathbf{\%}(*)$ & $\mathbf{p}$ \\
\hline Moradia próxima tráfico de drogas & $65,20 \%$ & 0,762 \\
Uso abusivo de álcool na família & $34,80 \%$ & 0,762 \\
Ameaças de expulsão de casa & $26,10 \%$ & 0,735 \\
Moradia em zona de criminalidade & $23,90 \%$ & 0,320 \\
Sentimento de culpa pela gravidez & & \\
na adolescência & $23,90 \%$ & 0,092 \\
Moradia em zona de prostituição & $19,60 \%$ & 0,716 \\
Uso abusivo de drogas na família & $17,40 \%$ & 0,700 \\
Uso abusivo de álcool ou drogas pelo & $13,00 \%$ & 0,213 \\
pai da criança & & \\
Problemas de conflito com a lei & $10,90 \%$ & 0,387 \\
na família & & \\
Problemas de conflito com a lei com & $10,90 \%$ & 0,144 \\
o pai da criança & $2,20 \%$ & 0,413 \\
Sofrer violências físicas & &
\end{tabular}

(*) com pontuações 3 (frequentemente) e 4 (sempre)

Tabela 6: Percentuais Fatores de Proteção na Escala FRIP

\begin{tabular}{lcc}
\hline \multicolumn{1}{c}{ Tema } & $\mathbf{\%}(*)$ & $\mathbf{p}$ \\
\hline $\begin{array}{l}\text { Manutenção de contato com o pai } \\
\text { da criança }\end{array}$ & $95,70 \%$ & 0,504 \\
Esperanças e sonhos para a vida & $93,50 \%$ & 0,999 \\
Apoio da família de origem & $87,00 \%$ & 0,999 \\
Relacionamento estável com o & & \\
companheiro & $84,80 \%$ & 0,999 \\
Percepção positiva da vida e bom humor & $82,60 \%$ & 0,999 \\
Apoio da família do pai da criança & $80,40 \%$ & 0,999 \\
Bom vínculo com a família do pai da & & \\
criança & $80,40 \%$ & 0,999 \\
Pai da criança trabalha & $80,40 \%$ & 0,999 \\
Bom relacionamento com vizinhos & $78,30 \%$ & 0,488 \\
Valorização da escola & $76,10 \%$ & 0,320 \\
Percepção positiva da adolescência & $76,10 \%$ & 0,032 \\
Bom vínculo com família de origem & $73,90 \%$ & 0,999 \\
Morar há mais de 2 anos no bairro & $71,70 \%$ & 0,746 \\
Morar próximo a parentes & $67,40 \%$ & 0,025 \\
Boa vinculação com escola & $67,40 \%$ & 0,341 \\
Facilidade de acesso a serviços de saúde & $63,00 \%$ & 0,235 \\
Frequentou escola nos últimos 4 meses & $60,90 \%$ & 0,037 \\
Ter crença religiosa & $52,20 \%$ & 0,080 \\
Ter opçóes para esportes & $43,50 \%$ & 0,999 \\
Ter apoio econômico & $39,10 \%$ & 0,541 \\
Ter atividades de lazer & $34,80 \%$ & 0,999 \\
Ter opções para atividades culturais & $17,40 \%$ & 0,700 \\
Trabalho com registro em carteira, & $15,20 \%$ & 0,999 \\
últimos 4 meses & & \\
\hline (*) com pontuçõ 3 (frequentemente) & & \\
\hline & &
\end{tabular}

(*) com pontuações 3 (frequentemente) e 4 (sempre) 
percentuais de respostas do grupo investigado (adolescentes gestantes mães) que assinalaram os graus 3 (frequentemente) ou 4 (sempre) para os itens da escala.

Os subgrupos de gestantes e mães investigadas apresentaram diferenças para cinco dentre 34 itens da escala (empregou-se o Teste exato de Fisher). Destes cinco, quatro itens foram relacionados a fatores de proteção e um de risco, a saber: "ter percepção positiva da adolescência" ( $88,9 \%$ das gestantes e $57,9 \%$ das mães), "morar próximo a parentes" (81,5\% das gestantes e $47,4 \%$ das mães), "ter frequentado escola nos últimos quatro meses" ( $74,1 \%$ das gestantes e $42,1 \%$ das mães), "ter crença religiosa" ( $40,7 \%$ das gestantes e $68,4 \%$ das mães), e "possuir sentimento de culpa pela gravidez ocorrida na adolescência" (33,3\% das gestantes e $10,5 \%$ das mães).

\section{DISCUSSÃO}

Os resultados do YSR não indicaram problemas psicológicos / comportamentais ou de competência social no grupo investigado. Embora não se tenha detectado diferença do ponto de vista estatístico, os menores escores de Competência obtidos pelas adolescentes mães e gestantes de classes econômicas mais pobres levantam a questão de que a pobreza exerça influência negativa no tocante ao desenvolvimento da Competência.

Quanto à avaliação de problemas psicológicos, apenas problemas externalizantes (de violação de regras) foram indicados em níveis limítrofes, e no subgrupo das adolescentes mães. Quando considerados por frequências de dados clínicos, queixas somáticas e de isolamento estiveram ausentes nas mães investigadas, da mesma forma que queixas de solidão foram pouco presentes nas gestantes (5\%) e totalmente ausentes no subgrupo das mães.

Estudo de Reis e Oliveira ${ }^{5}$ apontou que, pela percepção de adolescentes pobres, a com- panhia para a solidão é motivo importante para uma adolescente engravidar.

O estudo de Rocha e Silvares ${ }^{25}$ com 2248 jovens brasileiros indicou que, comparativamente, os adolescentes que apresentam menos problemas psicológicos (no caso os não encaminhados para clínicas escola de cursos de Psicologia) possuem maior pontuação na escala Desempenho Acadêmico do YSR.

No presente estudo, as adolescentes mães apresentaram menores escores nessa área de Desempenho Acadêmico quando comparadas às adolescentes gestantes.

São vários os trabalhos que destacam problemas de evasão escolar relacionados à gravidez na adolescência ${ }^{7,36-38}$ e a saída da escola pode ser mais presente em adolescentes mães do que em adolescentes gestantes.

Trabalho de Oliveira-Monteiro ${ }^{3}$ questiona a relação entre a evasão escolar de mães adolescentes com marcantes dificuldades para colocação dos filhos em creches. No presente estudo, os escores de faixa limítrofe do YSR no âmbito de Atividades (que englobam passatempos e esportes) obtidos pelas adolescentes mães parecem estar associados à maternidade, condição que pode afastar a adolescente de outras atividades sociais devido à dedicação ao bebê.

O abandono escolar de adolescentes grávidas e mães, especialmente aquelas mais pobres, agrava suas dificuldades econômicas com prováveis efeitos negativos na futura inserção no mercado de trabalho ${ }^{4,8,39-40}$. Por outro lado, segundo a $\mathrm{OMS}^{2}$, a pobreza influencia na probabilidade de uma jovem engravidar o que pode gerar um círculo vicioso, quando uma maternidade precoce vem a comprometer o rendimento escolar e o potencial econômico da mãe adolescente.

Resultados da escala FRIP mostraram que a escola foi valorizada por $76,1 \%$ do grupo investigado, com referências de frequência à escola (pelo menos até quatro meses antes da investigação) por $74 \%$ de gestantes e $42,1 \%$ das mães. 
Recentes estudos brasileiros ${ }^{33,41}$ destacam três tipos de fatores de proteção para crianças e adolescentes: atributos individuais, atributos familiares e fontes de apoio individual e institucional. Nesse sentido, a inserção na rede formal de educação pode ser fator de proteção para adolescentes, pela oportunidade de maior interação com pares e com adultos de confiança. A escola constitui ambiente de inúmeras relações face a face, próprias do chamado meso sistema $^{18}$ que podem constituir-se fatores de proteção ao desenvolvimento.

Outros indicativos de fatores de proteção e de risco puderam ser identificados no estudo, através da escala FRIP, como aqueles subjacentes a condições de desenvolvimento próprias de contextos familiares, de relação com o companheiro/pai da criança*, de referências a drogas e violência, além de possíveis recursos pessoais para resiliência.

Discutindo esses fatores com utilização de parâmetros de Bronfenbrenner ${ }^{18}$, primeiramente pode-se verificar que na maioria do grupo pesquisado houve indicativos de fatores de proteção pertinentes aos micro e meso contextos ambientais, aqueles próprios das relações face a face. Foram eles: a manutenção de contato com o pai da criança ( $95,7 \%$ do grupo), com indicativos de relacionamento estável $(84,8 \%)$, o apoio da família de origem ( $87 \%$ ) e da família do pai da criança $(80,4 \%)$, bom relacionamento com vizinhos $(78,3 \%)$, estabilidade de moradia $(71,7 \%)$ e proximidade de parentes $(67,7 \%)$.

Fatores de risco subjacentes a esses sistemas ambientais micro e meso foram indicados em menos da metade do grupo investigado, quanto a: uso abusivo de álcool e de drogas na família $(34,8 \%)$, e ameaças de expulsão de casa por conta da gravidez $(26,1 \%)$.

As referências a drogas e violência, como fatores de risco, estiveram também presentes em questões da escala FRIP especial- mente com respeito a: proximidade da moradia a locais de tráfico de drogas $(65,2 \%)$ e de prostituição (19,6\%); presença de criminalidade no local de moradia $(23,9 \%)$; além de vitimização com violência física (caso indicado por uma mãe adolescente investigada).

Esses são indicadores de fatores de risco que permeiam também o micro e o meso sistemas, mas que podem alcançar dimensões consideradas como de exo sistema. O exo sistema inclui aqueles contextos ambientais em que as próprias gestantes/mães adolescentes não estão presentes, mas que interagem com suas condições de desenvolvimento (adolescência/gravidez/maternidade) e podem ser por elas também influenciados. Destaque-se nesse sentido a proximidade da moradia a locais de tráfico de drogas como referência frequentemente ou sempre presente para mais da metade das gestantes (63\%) e das mães (68\%) investigadas.

Já o uso abusivo de álcool ou drogas pelo companheiro, indicado como fator de risco por $13 \%$ do grupo pesquisado, permeia dimensões de micro a exo sistemas, da mesma forma que problemas de conflito com a lei na família de origem e com o pai da criança ( $10,9 \%$ para ambos indicadores). $\mathrm{O}$ indicativo do pai da criança trabalhar (em 80,4\% do grupo estudado) sugere fator de proteção relativo a exo sistema, assim como a referência quanto a ter apoio econômico (39,1\% do grupo).

Por outro lado, algumas questões da escala FRIP (como esperanças e sonhos para a vida, percepção positiva da vida e bom humor, visão positiva da adolescência, e crença religiosa) podem ser recursos pessoais mais propícios para resiliência, conforme referências da literatura pertinente ${ }^{16,35}$. As esperanças e sonhos para a vida foram indicadas pela maioria do grupo investigado (93,5\%), assim como a referência de ter percepção positiva da vida e bom humor $(82,6 \%)$.

* A escala continha cinco itens acerca do "pai da criança" e sua família e um item sobre "companheiro", que poderia não ser o pai da criança. 
A referência sobre percepção positiva da adolescência foi diferente nos subgrupos pesquisados, com indicadores de maior presença dessa percepção positiva nas adolescentes gestantes $(88,9 \%)$ do que nas adolescentes mães $(57,9 \%)$.

O estudo de Oliveira-Monteiro ${ }^{3}$ indicou que algumas características de maternidade parecem sobrepor-se às da adolescência, no tocante ao desenvolvimento de adolescentes mães. As adolescentes mães podem se identificar mais com mulheres adultas, pelo papel de mãe, do que com mulheres adolescentes, como também indicado por Esteves e Menandro ${ }^{42}$, na questão do atributo da responsabilidade de mães adolescentes.

A afirmação de possuir crença religiosa foi mais frequente no subgrupo das adolescentes mães $(68,4 \%)$ em comparação às adolescentes gestantes $(40,7 \%)$, enquanto o sentimento de culpa pela gravidez ocorrida na adolescência, também dimensão pessoal investigada em item da escala FRIP, apareceu

\section{REFERÊNCIAS}

1. Brasil. Ministério da Saúde. Secretaria de Atenção à Saúde. Departamento de Ações Programáticas Estratégicas. Marco teórico e referencial da saúde sexual e reprodutiva de adolescentes e jovens. Brasília; Ministério da Saúde: 2006; 17-18.

2. WHO - World Health Organization. Adolescent pregnancy: a culturally complex issue. Bull World Health Organ. 2009;87:410-11.

3. Oliveira-Monteiro NR. Percursos da Gravidez na adolescência: estudo longitudinal após uma década da gestação. Psicol. Reflex. Crít. 2010;23(2):278-88.

4. Oliveira-Monteiro NR. Perfis de adolescentes mães após três anos e meio do nascimento do bebê: seguimento longitudinal mais referido por gestantes $(33,3 \%)$ do que por mães $(10,5 \%)$. Em contrapartida, a questão aberta da escala apresentou respostas muito comuns, de adolescentes mães e gestantes, indicativas da grande valorização da criança, nascida ou por nascer, como aquilo que era "o melhor de suas vidas".

As autorreferências de qualidades pessoais presentes especialmente nas adolescentes mães podem ser associadas a essa valorização da situação da maternidade.

Os diversos fatores de proteção com presença indicada nos contextos ambientais das adolescentes grávidas e mães pesquisadas podem estar associados com os níveis de competência identificados nessas jovens e também exercer influências protetivas importantes diante de riscos decorrentes da inserção e proximidade ambientais do tráfico de entorpecentes. Sugere-se a continuidade de estudos e aprofundamentos relativos a fatores de proteção e de risco associados ao desenvolvimento de adolescentes grávidas e mães.

de estudo psicossocial. Interação em Psicologia. 2008;12(2):291-97.

5. Reis AOA, Oliveira-Monteiro NR, Sexualidade e procriação na ótica de jovens de periferias sociais e urbana. Rev. bras. crescimento desenv. hum. 2007;17(2): 54-63.

6. Santos Junior JD. Fatores etiológicos relacionados à gravidez na adolescência: vulnerabilidade à maternidade. In: Schor N, Mota MSFT, Branco VC. Cadernos juventude, saúde e desenvolvimento. Brasília: Ministério da Saúde; 1999. p.223-29.

7. Chalem E, Mitsuhiro SS, Ferri CP, Barros MCM, Guinsburg R, Laranjeira R. Gravidez na adolescência: perfil sócio demográfico e comportamental de uma população da periferia de São Paulo, Brasil. Cad. Saúde Pública. 2007;23(1):177-89. 
8. Godinho RA, Schelp JRB, Parada CMGL, Bertoncello NMF. Adolescentes e grávidas: onde buscam apoio? Rev. latino-am. enfermagem. 2000;8(2):25-32.

9. Moffitt TE. E-Risk Study Team. Teen-aged mothers in contemporary Britain. J Child Psychol Psychiatry. 2002;43(6):727-42.

10. Kessler RC, Berglund PA, Foster CL, Saunders WB, Stang PE, Walters EE. Social consequences of psychiatric disorders II: Teenage parenthood. Am J Psychiatry. 1997;154 (10):1405-11

11. Sapienza G, Pedromônico MRM. Risco, proteção e resiliência no desenvolvimento da criança e do adolescente. Psicologia em Estudo. 2005;10(2): 209-16.

12. Lindstrom B. O significado de resiliência. Adolesc. Latinoam. 2001; 2(3): 133-37.

13. Poletto M, Koller SH, Dell'Aglio DD. Eventos estressores em crianças e adolescentes em situação de vulnerabilidade social de Porto Alegre. Ciência e saúde coletiva. 2009;14(2):455-66.

14. Sapienza G. Competência Social, práticas educativas parentais e rendimento académico de adolescentes. [Tese]. São Paulo: Universidade Federal de São Paulo; 2006; p.13-17.

15. Aznar-Farias M, Oliveira-Monteiro NR. Reflexões sobre pró-socialidade, resiliência e psicologia positiva. Rev. Bras.Ter. Cogn. 2006;2:39-46.

16. Dell'aglio DD, Koller SH, Yunes MAM. Resiliência e Psicologia Positiva: interfaces do risco à proteção. São Paulo: Casa do Psicólogo; 2006.

17. Cerqueira-Santos E, Paludo SS, Schirò EDB, Koller SH. Gravidez na adolescência: análise contextual de risco e proteção. Psicol. estud. 2010;15(1):72-85.

18. Bronfrenbrenner UA. Ecologia do desenvolvimento humano. Porto Alegre: Artmed; 2001.

19. De Antoni C, Koller SH. A visão de família entre as adolescentes que sofreram vio- lência intrafamiliar. Estud. psicol. 2000;5(2):347-81.

20. De Antoni C, Koller SH. Vulnerabilidade e resiliência familiar: um estudo com adolescentes que sofreram maus-tratos intrafamiliares. Psicologia em Estudo. 2000;31(1):39-66.

21. Yates Y, Selaman R. Social Competenc in school: towards an integrative developmental model for intervention. Developmental Review. 1989; 9: 64-100.

22. Trianes MV, Muñoz AM, Jiménez M. Competencia Social: su educación y tratamiento. Madri: Pirámide; 1997.

23. Achenbach TM, Edelbrock C. The manual for the youth self-report and profile. Burington: University of Vermont; 1987.

24. Dell'aglio DD, Hutz CS. Depressão e desempenho escolar em crianças e adolescentes institucionalizados. Psicol. reflex. crít. 2004;17(3):351-57.

25. Rocha, MM, Silvares EFM. Competências de jovens brasileiros: fator de proteção para problemas comportamentais. In: Actas do VII Simpósio Nacional de Investigação em Psicologia. Portugal: Universidade do Minho; 2010; p. 1162-71

26. Associação Brasileira de Empresas de Pesquisa (ABEP). Critério de classificação econômica Brasil. São Paulo: Associação Brasileira de Empresas de Pesquisa; 2003; p. 1-3.

27. Achenbach TM, Rescorla LA. Mental Health practitioners' guide for the Achenbach System of Empirically Based Assessment (ASEBA). 4th ed. Burlington: University of Vermont - Research Center for Children, Youth, \& Families; 2004.

28. Rocha, MM. Auto-avaliação de competências e problemas de comportamento entre adolescentes brasileiros: um estudo de validação do "Inventário de Auto-Avaliação para Jovens" (YSR/2001). [Projeto de Qualificação para Mestrado]. São Pau- 
lo: Instituto de Psicologia da Universidade de São Paulo; 2009; p.42-83.

29. Dias MDF. Adolescentes infratores e não infratores: uma análise comparativa através do CBCL e do YSR. [Dissertação]. São Paulo: Universidade Federal de São Pau1o; 2001. p. 37-39.

30. Massola GM, Silvares EFM. A percepção do distúrbio de comportamento infantil por agentes sociais versus encaminhamento para atendimento psicoterapêutico. Rev. Inter Psicologia. 2005;39(1):139-50.

31. Melo MHS, Silvares EFM. Grupo cognitivo-comportamental com famílias de crianças com déficits em habilidades sociais e acadêmicas. Temas Psicol. 2003;11(2):122-33.

32. Shoen-Ferreira TH. A adolescência e a formação da identidade: uma proposta de avaliação e intervenção. [Tese]. São Pau1o: Universidade Federal de São Paulo; 2007. p. 81-83; 107-15.

33. Pesce R, Assis SG, Santos N, Oliveira RV. Risco e proteção: um equilíbrio protetor de resiliência. Psic Teoria Pesq. 2004;20(2):135-43.

34. Pesce RP, Assis SG, Avanci JQ, Santos NC, Caravalhaes R. Adaptação transcultural, confiabilidade e validade da escala de resiliência. Caderno de Saúde Pública. 2005;21(2):436-48.

35. Poletto RC, KOLLER SH. Resiliência: uma perspectiva conceitual e histórica. In: Dell'aglio DD, Koller SH, Yunes MAM (org.). Resiliência e psicologia positiva: interfaces do risco à proteção. São Paulo: Casa do Psicólogo; 2006.
36. Heilborn ML, Salem T, Rohden F, Brandão L, Knauth D, Víctoria C et al. Aproximações socioantropológicas sobre a gravidez na adolescência. Horiz. antropol. 2002; 17(8):13-45.

37. Traverso-Yepez MA, Pinheiro VS. Gender socialization and adolescence. Rev. Estud. Fem. 2005; 13(1):147-62.

38. Rosa AJ, Reis AOA, Tanaka ACA. Gestações Sucessivas na Adolescência. Rev Bras Crescimento Desenvolv Hum. 2007;17(1):165-72.

39. Cannon LRC. Prefácio. In: Vieira EM, Fernandes MEL, Bailey P, McKay A. (Orgs.). Seminário Gravidez na Adolescência. Rio de Janeiro: Ministério de Saúde. Family Health International/Associação Saúde da Família; 1998.

40. Aquino EML, Heilborn ML, Knaut D, Bozon M, Almeida MC, Araújo J, Menezes G. Adolescência e reprodução no Brasil: a heterogeneidade dos perfis sociais. Cad Saúde Pública. 2003;19(Sup 2):S377-S88.

41. Maia JMD, Williams LCA. Fatores de risco e fatores de proteção ao desenvolvimento infantil: uma revisão da área. Temas em Psicologia. 2005;13(2):91-103.

42. Esteves JR, Menandro PRM. Trajetórias de vida: repercussões da maternidade adolescente na biografia de mulheres que viveram tal experiência. Estud. psicol. 2005;10(3): 363-70.

Recebido em: 30/jan./2011 Modificado em 12/mar./2011 Aceito em 30/mar./2011 